\title{
Fundamentals of Deep Neural Networks - Application Based on MLPs
}

\author{
José L. Barretto*, Romis Attux.
}

\begin{abstract}
Deep Neural Networks have become one of the center pieces in the field of machine learning, mostly due to accuracy, practical applications and efficiency. These algorithms have proven to be the state of the art in solving tasks such as regressions, clustering and image classification. The purpose of this project was to study and implement deep learning applications using one of the most simple and powerful networks: the multilayer perceptron. Therefore, the project started with a theorical approach and built towards the design of an MLP-based deep neural network capable of classifying hand-written digits.
\end{abstract}

\section{Key words: \\ Deep Neural Networks, Machine Learning, Computational Intelligence}

\section{Introduction}

The multilayer perceptron (MLP) is a paradigmatic neural network model and an important model in deep learning. An MLP is a combination of mathematical functions that map some set of input values onto one or more outputs [1]. The output of this model can be compared to a set of desired values / labels to calculate a cost, which is used, with the aid of the backpropagation algorithm, to modify the parameters of the model.

This training strategy is widely used in practical applications of deep learning, and, when properly used (that is, avoiding underfitting or overfitting of the inputs), can provide accurate results for common computational tasks.

The goal of this research was to utilize a multilayer perceptron model to correctly classify handwritten digits from the MNIST dataset [2].

\section{Results and Discussion}

The defined computational task was to correctly classify images of hand-written digits belonging to the MNIST dataset [2], which contains 60000 training examples and 10000 testing examples. With that in mind, an MLP deep neural network model with 5 hidden layers and a softmax output layer was designed. The used activation function was the ReLU.

The testing phase consisted of varying the network's hyperparameters, training and, finally, verifying the model's accuracy. The parameters that had more impact in the model were the learning rate (where a momentum was applied to optimize the parameter value) and the number of neurons in each hidden layer. With the aid of the open-source machine learning framework PyTorch, the models were implemented in a Python environment, and trained on an Nvidia GeForce 940MX GPU.

At the end of the testing phase, a reasonable parameter choice was that of 500 neurons for each hidden layer and a learning rate of 0.01 with 0.8 momentum. The resulting model reached an accuracy of approximately $97 \%$, a value that levels with results such as those stated by LeCun, Y. [2].

The network's cost was calculated through the negative log likelihood loss, and used by the softmax layer to generate a likelihood estimation of input-label mapping as an output. A stochastic gradient descent (SGD) algorithm with a batch learning approach was used for the model's training, that is represented in Image 1.
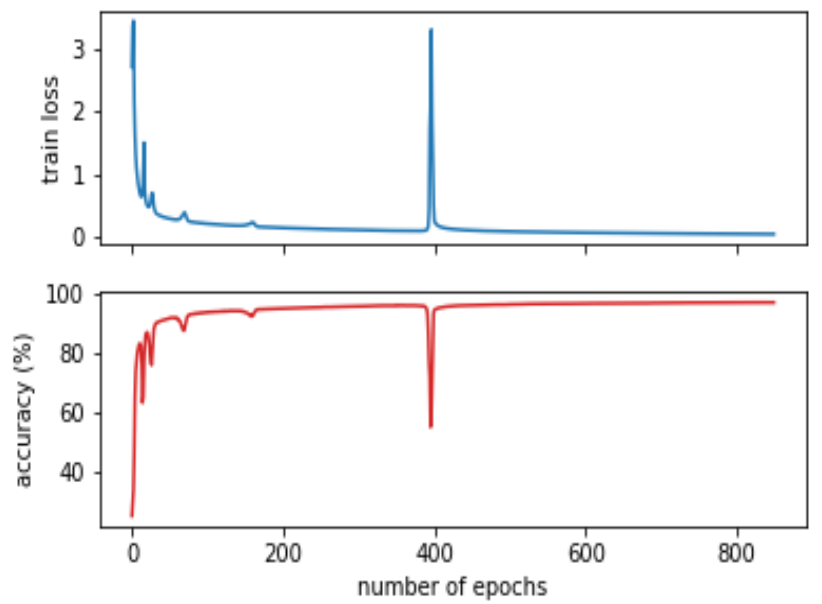

Image 1. Network's learning curve (Above: training loss negative log likelihood. Below: model accuracy).

\section{Conclusions}

This research project was very important to the student's introduction to the study of deep neural networks. It covered the basic underlying concepts that are very common in the field of machine learning, such as linear algebra, probability, information theory and numerical computation. These concepts were further utilized by the student to design and test a deep MLP network model to solve a classic computational task, the classification of hand-written digits. This implementation was followed by the optimization of the network's parameters to achieve near state-of-the-art accuracy.

\section{Acknowledgement}

The student wishes to express his gratitude to professor Romis R.F. Attux for the guidance and support and to the CNPq/PIBIC for the financial support.

\footnotetext{
${ }^{1}$ Goodfellow, I.; Bengio, Y.; Courville, A. Deep Learning, 2016.

2 LeCun, Y. The MNIST Database of Handwritten Digits. Available at: http://yann.lecun.com/ exdb/mnist/. Last accessed in: 09/07/2019.
} 